\title{
LOS RETOS DEL DERECHO AL TRABAJO Y AL OCIO DE LAS MUJERES EN ECUADOR*
}

\author{
THE CHALLENGES OF WOMEN'S RIGHT TO WORK \\ AND LEISURE IN ECUADOR
}

\section{LES DÉFIS DU DROIT DES FEMMES AU TRAVAIL ET AUX LOISIRS EN ÉQUATEUR}

\author{
Adriana MORA BERNAL ** \\ Ximena RON ERRÁEZ ${ }^{* * *}$ \\ Javier ÁVILA LARREA*** \\ Marcela SÁNCHEZ ${ }^{* * * * *}$
}

\begin{abstract}
RESUMEN: El presente artículo se propone analizar la desigualdad que sufren las mujeres en el ejercicio de los derechos humanos al trabajo y al ocio en el Ecuador. En cuanto a la metodología, la presente investigación utilizó el análisis económico del derecho, pretendiendo determinar los efectos del ejercicio del derecho al trabajo y al ocio por parte de las mujeres. El estudio se centró en estos derechos sobre la base de información disponible en el Instituto Nacional de Estadísticas y Censos y el Índice Global sobre Brechas de Género del Foro Económico Mundial. Con estos datos se evidenció la situación de desigualdad de las mujeres respecto a salarios, empleo pleno, desempleo y ocio en el Ecuador, a pesar de contar con normas internacionales y nacionales que protegen sus derechos fundamentales. Situación que se muestra mucho más compleja con la llegada de la pandemia, pero que a su vez trae el desafío por
\end{abstract} de 2021.

Recibido el 11 de noviembre de 2020 y aceptado para su publicación el 15 de marzo

** Docente e investigadora en la Universidad Católica de Cuenca.

*** Abogada litigante. Docente de posgrado e investigadora en la Universidad de Especialidades Espíritu Santo.

**** Docente e investigador en la Universidad Católica de Cuenca.

***** Docente e investigadora en la Universidad Católica de Cuenca.

\footnotetext{
T) Revista Latinoamericana de Derecho Social Núm. 33, julio-diciembre de 2021, pp. 41-68
} 
parte de los Estados a generar empleo tomando en cuenta los aspectos económicos y ambientales.

Palabras clave: derechos de la mujer, derecho al trabajo, desigualdad social, igualdad de oportunidades, ocio.

ABSTRACT: This article aims to analyze the inequality suffered by women in the exercise of the human rights to work and leisure in Ecuador. In terms of methodology, this research used the economic analysis of the right to determine the effects of the exercise of the right to work and leisure by women. The study focused on these rights based on information available from the National Institute of Statistics and Census and the Global Gender Gap Index of the World Economic Forum. These data showed the unequal situation of women concerning salaries, full employment, unemployment, and leisure in Ecuador, despite the existence of international and national norms that protect their fundamental rights. This situation is much more complex with the arrival of the pandemic, but at the same time brings the challenge for the States to generate employment considering economic and environmental aspects.

Keywords: women rights, right to employment, social inequality, equal opportunity, leisure.

RÉSUMÉ: Cet article vise à analyser les inégalités subies par les femmes dans l'exercice des droits humains au travail et aux loisirs en Équateur. En ce qui concerne la méthodologie, cette recherche a utilisé l'analyse économique du droit, procurant de déterminer les effets de l'exercice du droit au travail et aux loisirs par les femmes. Pour analyser ces droits, l'étude a utilisé les informations de l'Institut National des Statistiques et des Recensements et l'Indice Mondial de l'écart entre les genres du Forum Économique Mondial. Ces données mettent en évidence la situation inégale des femmes en termes de salaire, plein emploi, chômage et loisirs en Équateur, malgré les normes internationales et nationales qui protègent leurs droits fondamentaux. Ceci est d'autant plus complexe avec l'arrivée de la pandémie, ce qui à son tour pose le défi des États de créer des emplois en tenant compte des aspects économiques et environnementaux.

Most-clés: droits des femmes, droit au travail, inégalités sociales, égalité d'opportunités, loisir. 
SuMARIO: I. Introducción. II. Los derechos humanos de las mujeres. III. La igualdad de género en el derecho ecuatoriano. IV. El derecho al trabajo y al ocio. Dos temas relevantes en la igualdad de género en Ecuador. V. Discusión y resultados. VI. Conclusiones. VII. Bibliografía.

\section{INTRODUCCIÓN}

\section{工}

a pandemia ha provocado grandes impactos económicos a nivel mundial, y para América Latina y el Caribe éstos se presentaron en un momento de debilidad y vulnerabilidad macroeconómica, ${ }^{1}$ profundizando las desigualdades y exacerbando los impactos hacia las mujeres y niñas únicamente por su sexo. ${ }^{2}$ Naciones Unidas ha identificado que serán las mujeres y niñas quienes reciban mayoritariamente sus efectos, ya que generalmente ganan menos, ahorran menos, tienen trabajos inseguros y viven cerca de la pobreza. ${ }^{3}$

La COVID-19 ha traído devastadoras consecuencias sociales y económicas, debiendo los gobiernos limitar los efectos adversos que sufrirá el mercado de trabajo. ${ }^{4}$ Este reto estará latente más allá de la posibilidad de que una vacuna esté disponible a inicios o mediados de 2021 en los países de la región. ${ }^{5}$ "Este escenario provocará la mayor crisis económica y social de la región de las últimas décadas, que afectará de manera muy negativa al empleo, la lucha contra la pobreza y la reducción de la desigualdad, compromisos asumidos en la Agenda 2030 para el Desarrollo Sostenible". En este marco, el Sistema de Naciones Unidas priorizó el reporte de avance y desafíos de los objetivos 4, 8, $10,13,16$ y 17, siendo los más importantes para este trabajo el objetivo 8 y 10 que abordan el empleo digno y crecimiento económico, así como la reducción de la desigualdad.

1 CEPAL/OIT, Coyuntura Laboral en América Latina y el Caribe. El Trabajo en Tiempos de Pandemia: Desafíos frente a la Enfermedad por Coronavirus (COVID-19), Santiago, 2020 , p. 7.

2 Naciones Unidas, The Impact of COVID-19 on Women, 2020; disponible en https:// www.unwomen.org/-/ media/ headquarters/attachments/sections/library/publications/2020/policy-briefthe-impact-of-covid-19-on-women-en.pdf?la $=$ en 6 vs $=1406$.

3 Idem.

4 OIT, Brindar Apoyo a los Ingresos y al Empleo en Tiempos de la COVID-19: Articular las Transferencias en Efectivo con Políticas Activas del Mercado de Trabajo (PAMT), p. 1.

5 OIT, Panorama Laboral 2020. América Latina y el Caribe, 2020, p. 58.

6 CEPAL/OIT, op. cit., p. 7. 
Así también, en 2015 la OIT adoptó las “Directrices de política para una transición justa hacia economías y sociedades ambientalmente sostenibles para todos", que sitúa como eje principal el trabajo decente. La agenda de transición justa permite identificar la relación entre el empleo y el medio ambiente y los impactos que se evidencian entre éstos. Como información relevante, en América Latina y el Caribe, alrededor de 64 millones de empleos dependen de los servicios ecosistémicos. ${ }^{7}$

En consecuencia, los efectos de la pandemia conllevan muchos desafíos para los gobiernos, pero también nuevas propuestas deben ser analizadas por los Estados para la recuperación postcovid-19; estas últimas tienen que dirigirse hacia la creación de empleos verdes y un crecimiento sostenible, como lo sugiere la OIT. En esta línea argumentativa, el secretario general de Naciones Unidas hace un llamado a que los países trabajen para que los fondos que se gastarán en la recuperación de los efectos del coronavirus generen empleos y negocios que permitan un crecimiento sostenible. ${ }^{8}$

Entonces, por un lado, nos encontramos frente al desafío de plantear la posibilidad de abordar los impactos económicos del coronavirus, y, por otra parte, la promoción de la descarbonización y la protección ambiental. En este sentido, la agenda de transición justa y empleo verde ha identificado sectores claves como la bioeconomía, energía y minería, turismo, movilidad y transporte, construcción, residuos y economía circular y manufactura. Sectores que para el Ecuador representan el $58 \% \%^{9}$ y que se han visto afectados por la COVID-19, pero que al mismo tiempo podrían convertirse en una potencial fuente de creación de empleos.

En conclusión, antes de la pandemia, los derechos laborales de las mujeres se enfrentaban a grandes retos, y en el contexto actual "en América Latina y el Caribe la COVID-19 ha ocasionado una devastación sin precedente en el mundo del trabajo con estimaciones de pérdida de 47 millones de empleo en la región". ${ }^{10}$ Por ello, la presente investigación plantea analizar la situación de las mujeres respecto a las brechas existentes en salarios, empleo, subempleo, desempleo y ocio, antes y durante la pandemia, así como los retos a los que se enfrenta el mercado laboral, el cual debe evitar la permanencia de las brechas

7 OIT y BID, Jobs in a Net-Zero Emissions Future in Latin America and the Caribbean, 2020, p. 27; disponible en http://dx.doi.org/10.18235/0002509.

8 Sánchez, Ana Belén y Torres, Braulio, Una recuperación verde y justa en América Latina y el Caribe: una perspectiva desde el mundo del trabajo, OIT, 2020, p. 2.

9 Ibidem, p. 7.

10 Ibidem, p. 3. 
entre hombres y mujeres; aquí, el gobierno debe mejorar las condiciones de trabajo decente dentro de un marco de diálogo social, para que nadie se quede atrás y los países creen los empleos y la estructura productiva necesaria para la gestión sostenible de los recursos naturales. ${ }^{11}$

\section{LOS DERECHOS HUMANOS DE LAS MUJERES}

Cuando hablamos de derechos humanos, nos referimos a unos derechos que — si bien teóricamente han incluido tanto a hombres como a mujeres-, en la práctica, bajo su máscara de supuesta neutralidad, han excluido indefectiblemente a las mujeres. ${ }^{12}$ Por lo que, "desde el movimiento de las mujeres y feministas, así como de distintos grupos sociales, se han hecho muchas críticas a los derechos humanos, poniendo en evidencia su falta de universalidad". ${ }^{13}$ En 1991, con la campaña mundial "Los derechos de las mujeres son derechos humanos" se abordó la existencia de brechas entre la teoría y la práctica de derechos humanos, resultando insuficiente el concepto de igualdad formal ante la ley, emergiendo la necesidad de transitar a una igualdad material.

La Declaración Universal de Derechos Humanos, adoptada en París en 1948 en el artículo 1, afirmó que "todos los seres humanos nacen libres e iguales en dignidad y derechos". El reconocimiento, aplicación y ejercicio de los derechos de las personas, de acuerdo con lo que señala ese mismo cuerpo normativo, no responde a distinción alguna de raza, color, sexo, idioma, religión, opinión política o de cualquier otra índole, origen nacional o social, posición económica, nacimiento o cualquier otra condición. ${ }^{14}$

Más de siete décadas después de la adopción de la Declaración Universal de Derechos Humanos, casi todos los instrumentos internacionales en esta materia insisten en la igualdad entre las personas e incluso realizan menciones expresas a la igualdad entre los sexos. Ejemplos de esto se encuentran contenidos en el Pacto Internacional de Derechos Civiles y Políticos y el Pacto Internacional de Derechos Económicos, Sociales y Culturales (PIDESC), de 1966, los cuales consagran el compromiso de los Estados Parte de garantizar a hombres y mujeres la igualdad en el goce de los derechos.

11 Ibidem, p. 2.

12 Navarro, Marysa, "Los derechos humanos de las mujeres", Foro de la Filantropía y Mujeres, México, 1993, p. 217.

13 Eusko Jaurlaritza Gobierno Vasco et al., Tribunal Internacional de Derechos de las Mujeres. Viena +20. Euskalherria 2013, Mugarikgab, 2013, p. 11; disponible en wmw.mugarikgabe.org.

14 Naciones Unidas, Declaración Universal de Derechos Humanos, 1948. 
En función del fortalecimiento de la igualdad elevada a categoría de principio general del derecho, se han desarrollado, además, instrumentos internacionales de derechos humanos enfocados exclusivamente en erradicar la discriminación por sexo, como una de las formas específicas de transgresión al principio de igualdad. Tal es el caso de la Convención sobre la Eliminación de Todas las Formas de Discriminación Contra la Mujer y su Protocolo Facultativo, adoptados en 1979 y 1999, respectivamente, por la Asamblea General de las Naciones Unidas. ${ }^{15}$

Nótese que en el derecho internacional de los derechos humanos el principio de igualdad opera casi siempre junto al principio de no discriminación, mostrado como su consecuente inevitable. Sobre esta base, se fundamenta la prohibición de un trato desigual o diferente hacia ciertas personas por condiciones personales o sociales. Estos principios han sido ratificados por la Declaración y Programa de Acción de Viena, adoptada durante la Conferencia Mundial de Derechos Humanos de 1993, estableciendo en sus párrafos 5 y 18 que los Estados deben promover y proteger los derechos humanos de la mujer y la niña en condiciones de igualdad, erradicando todas las formas de discriminación. ${ }^{16}$

Precisamente en este sentido, la Corte Interamericana de Derechos Humanos señala, en su Opinión Consultiva OC-18/03 de 17 de septiembre de 2003, que: "la no discriminación, junto con la igualdad ante la ley y la igual protección de la ley a favor de todas las personas, son elementos constitutivos de un principio básico y general relacionado con la protección de los derechos humanos". Ahora bien, la evidente importancia en el contexto internacional de los principios de igualdad y no discriminación, especialmente en cuanto a la condición relacionada con el sexo y el género, se replica también en las legislaciones domésticas. Casi todos los países cuentan con normas jurídicas que garantizan la igualdad de derechos entre hombres y mujeres. Todo soportado en la noción de dignidad de las personas, que restringe actuaciones y tratos con privilegio o con hostilidad hacia y en contra de determinados grupos.

A pesar de que los principios de igualdad y no discriminación se encuentran contenidos en distintos instrumentos internacionales, desafortunadamente, la

15 Asamblea General de Naciones Unidas, Convención sobre la Eliminación de Todas las Formas de Discriminación Contra la Mujer, 1979. Adhesión por la Asamblea General en su resolución 34/180, de 18 de diciembre de 1979, con entrada en vigor el 3 de septiembre de 1981, de conformidad con el artículo 27 (1).

16 Conferencia Mundial de Derechos Humanos, Declaración y Programa de Acción de Viena, 1993. 
realidad sigue demostrando que, a pesar de existir esta prohibición, aún existen actos que constituyen discriminación contra las mujeres, justificados debido a la cultura, la religión o la tradición. ${ }^{17}$ Aún no se ha llegado a alcanzar el resultado esperado de igualdad entre hombres y mujeres, esto debido a que existen diferentes factores que hacen que se postergue el disfrute pleno de los derechos de las mujeres. ${ }^{18}$ Por ello, "es necesario que los Estados deconstruyan las nociones esencialistas de la cultura y revisen críticamente las propias prácticas culturales sexistas que discriminan a las mujeres y que frecuentemente son normalizadas o justificadas". ${ }^{19}$

\section{LA IGUALDAD DE GÉNERO \\ EN EL DERECHO ECUATORIANO}

Para contextualizar la situación de los derechos de las mujeres en Ecuador, es necesario hacer referencia al proceso de constitucionalización del orden jurídico, que ha tenido a lugar en países europeos y latinoamericanos; siendo un elemento característico, el reconocimiento de la fuerza normativa vinculante de la Constitución. ${ }^{20}$ En este sentido, Ecuador, con la Constitución del 2008, se constituyó en un Estado constitucional de derechos y justicia, y tiene como deber primordial garantizar sin discriminación alguna el efectivo goce de los derechos establecidos en la Constitución y en los instrumentos internacionales (artículo 1o.), transitando de un Estado de derecho a un Estado constitucional de derechos y justicia.

Bajo estas consideraciones, a lo largo de su texto en la Constitución del 2008, se consagran derechos de no discriminación a favor de los ciudadanos. El artículo 3o. numeral 1 dispone que se debe "garantizar sin discriminación alguna el efectivo goce de los derechos establecidos en la Constitución y en

17 Facio, Alda y Victoria, Anya, "Los derechos culturales y los derechos de las mujeres son Derechos Humanos y como tales, deben ser gozados en igualdad", Revista Europea de Derechos Fundamentales, núm. 29, 2017, p. 69; disponible en bttps://dialnet.unirioja.es/servlet/ articulo? codigo $=6143999$.

18 Gil Acevedo, Juan Carlos, "Ruta hacia la dignificación laboral de las madres comunitarias y sustitutas en Villavicencio, Colombia”, Estudios de Derecho, núm. 72, 2015, p. 169; disponible en https:// doi.org/10.17533/udea.esde.v72n160a07.

19 Facio y Victoria, op. cit., p. 69.

20 Aguiló Regla, Josep, "Positivismo y postpositivismo: dos paradigmas jurídicos en pocas palabras", Doxa. Cuadernos de Filosofía del Derecho, núm. 30, 2007, p. 666; disponible en bttps:/ / doi.org/10.14198/doxa2007.30.55. 
los instrumentos internacionales, en particular la educación, la salud, la alimentación, la seguridad social y el agua para sus habitantes". Esto en concordancia con el artículo 66 numeral 4 que reconoce y garantiza a las personas el derecho a la igualdad formal y material y la no discriminación. Así como la igualdad formal y material consagrada en el artículo 11 numeral 2 de la Constitución de la República, que establece el derecho de todas las personas de gozar de los mismos derechos, deberes y oportunidades.

En concordancia con lo anterior, antes de la vigencia de la Constitución mencionada, los planes nacionales de desarrollo realizaron una mirada integradora, basada en un enfoque de derechos, que tiene como ejes la sostenibilidad ambiental y las equidades de género, generacional, intercultural y territorial. Bajo esta misma orientación, en el Plan Nacional para el Buen Vivir 20092013, se consolidó el enfoque de género como uno de los ejes transversales. Asimismo, el Plan Nacional de Desarrollo 2017-2021. Toda una Vida, plantea la reducción de los niveles de inequidad socioeconómica y de género. ${ }^{21}$

En este sentido, la Asamblea General de Naciones Unidas fija como plan para conseguir los objetivos de desarrollo sostenible a la Agenda 2030 de Desarrollo Sostenible, la cual indica que no es posible realizar todo el potencial humano y alcanzar el desarrollo sostenible si se sigue negando a la mitad de la humanidad el pleno disfrute de sus derechos humanos y sus oportunidades. ${ }^{22}$ Asimismo, la Agenda Regional de América Latina y El Caribe identifica cinco enfoques propositivos relacionados a los derechos de las mujeres: igualdad de género, derechos humanos de las mujeres, interseccionalidad e interculturalidad, democracia paritaria, representativa, participativa y laicidad, y desarrollo sostenible e inclusivo. ${ }^{23}$

En lo correspondiente al derecho humano al trabajo, éste se encuentra consagrado en la Declaración Universal de los Derechos Humanos, en su artículo 23, según el cual toda persona tiene derecho al trabajo, a la libre elección de su trabajo, a condiciones laborales equitativas y satisfactorias, y a la protección contra el desempleo, ${ }^{24}$ que le asegure, así como a toda su familia, una existencia conforme a la dignidad humana que será completada, en caso necesario, por cualesquiera otros medios de protección social. El Comité de Derechos Económicos, Socia-

21 Instituto Nacional de Estadísticas y Censos, Atlas de Género, 2018; disponible en https:// www.ecuadorencifras.gob.ec/documentos/web-inec/Bibliotecas/Libros/Atlas_de_Genero_Final.pdf.

22 Asamblea General de Naciones Unidas, Transformar nuestro Mundo. La Agenda 2030 para el Desarrollo Sostenible.

23 Instituto Nacional de Estadísticas y Censos, op. cit., p. 13.

24 Naciones Unidas, Declaración..., cit. 
les y Culturales, detalla a cada uno de los Estados cómo debe garantizar y proteger el derecho al trabajo, e indica los requisitos esenciales e interdependientes, como el de la disponibilidad, accesibilidad, la aceptabilidad y calidad.

En el caso ecuatoriano, la Constitución de la República, al ser un Estado constitucional de derechos y justicia, fija como uno de los objetivos del régimen de desarrollo la generación de trabajo digno y estable, y otros derechos relacionados al trabajo que están debidamente garantizados y que se derivan de los convenios y tratados ratificados por el Estado. Sin embargo, aún persiste la "reclusión de las mujeres en el espacio doméstico y su exclusión del ámbito laboral", ${ }^{25}$ impidiendo su realización como seres humanos tanto en su dimensión personal, así como económica. Esto a pesar de que la norma constitucional establece al trabajo como un deber social y como derecho económico, fuente de realización personal y base de la economía.

En este marco, es importante no perder de vista que a las mujeres se les ha negado el pleno goce del principio de igualdad, tanto en lo que se refiere a su dimensión formal como material. En la normativa, esta distinción se encuentra recogida en la Constitución ecuatoriana en el artículo 11.2 y artículo 66.4; y a nivel de jurisprudencia, la sentencia núm. 292-16-SEP-CC, emitida en el caso núm. 0734-13-EP por la Corte Constitucional del Ecuador, indica que la igualdad formal implica que ante el sistema jurídico todas las personas deben tener un trato igualitario; mientras que la igualdad material se refiere a un análisis de la realidad de la persona. Esto significa que "la igualdad formal entre los sexos se ha incorporado progresivamente al ordenamiento jurídico ecuatoriano, sin embargo, la igualdad sustancial está en proceso; así lo demuestran la subsistencia de realidades sociales desiguales". ${ }^{26}$

De esta manera, según asevera la Corte Constitucional, las mujeres tienen aún que enfrentar una dura realidad relacionada con la falta de una plena igualdad material o sustancial que abarca el propósito constitucional de terminar con la histórica situación de inferioridad que soportan, puesto que las mujeres tienen que luchar contra un statu quo que históricamente se expresa en marginación económica, política, jurídica y social. ${ }^{27}$ Además,

25 Caamaño Rojo, Eduardo, "Mujer y trabajo: origen y ocaso del modelo del padre proveedor y la madre cuidadora", Revista de Derecho, núm. 34, 2010, p. 186; disponible en https:// doi. org/10.4067/S0718-68512010000100005.

26 Corte Constitucional del Ecuador, Sentencia núm. 292-16-SEP-CC emitida en el caso núm. 0734-13-EP.

27 Valenzuela Reyes, María Delgadina, "Evolución legislativa sobre los derechos e igualdad jurídica de la mujer en México”, Revista Latinoamericana de Derecho Social, núm. 10, 2010, p. 343. 
cabe señalar que "la discriminación basada en el sexo, así como su incidencia, varía con el tiempo y según la estructura y la dinámica de los mercados laborales". ${ }^{28}$

$\mathrm{El}$ anterior razonamiento resulta congruente si se piensa que los principios de igualdad y no discriminación se aplican, principalmente, frente a grupos que han sido histórica y estructuralmente discriminados, como es el caso de las mujeres. Resultando la discriminación, como se vio supra, en una negación de la dignidad de una persona o grupo; de ahí que se muestre la relación entre el principio de igualdad y el principio de dignidad, siendo que la transgresión al primero deriva siempre en una vulneración del segundo.

No se trata entonces, únicamente, de lograr que las mujeres sean iguales a los hombres ante la ley, eliminando por ejemplo normas jurídicas aún subsistentes que las excluyen de la titularidad o el ejercicio de ciertos derechos; sino también, que se reconozca realmente su dignidad plena, en función de erradicar las desigualdades sustanciales, palpables en la realidad más allá de lo que dice la ley.

En este contexto, el empoderamiento económico de las mujeres es un requisito sine qua non para el desarrollo económico; al respecto, ONU Mujeres sostiene que las mujeres han de poder transformar sus propias vidas, así como sus comunidades al participar en los procesos y espacios que afectan su independencia económica (mercado laboral, mercados de productos y servicios y los eslabonamientos globales de dichos mercados). ${ }^{29}$

Es preciso indicar que "las relaciones entre la economía y las mujeres es una tarea que cobra una creciente relevancia y provoca cada vez mayor interés", 30 puesto que resulta difícil pensar en igualdad entre hombres y mujeres, así como un desarrollo sostenible, si no se incorpora a las agendas públicas la igualdad de oportunidades. Aunque en varios países se avanza hacia la paridad de género como señala el Índice Global sobre Brechas de Género, a menudo es a un ritmo lento, siendo la segunda brecha más grande la de participación y oportunidad económica, ocupando el Ecuador el puesto 74 de 153 países.

\footnotetext{
28 Tomei, Manuela y Vega-Ruiz, María Luz, "La discriminación de la mujer en el lugar de trabajo. Nuevas tendencias en materia de discriminación por motivos basados en la maternidad y el acoso sexual", Revista Latinoamericana de Derecho Social, núm. 4, 2007, p. 150; disponible en http:/ / biblio.juridicas.unam.mx/revista/pdf/DerechoSocial/4/art/art8.pdf.

29 Qué Hacemos. Empoderamiento Económico, ONU Mujeres, América Latina y el Caribe; disponible en https:/ / lac.unwomen.org/es/que-bacemos/empoderamiento-economico.

30 Carcedo, Ana et al., Apertura económica, género y pobreza en el istmo centroamericano, 2009, p. 7; disponible en http:// www.gemlac.org/attachments/article/313/Informe septiembre 2009.pdf, consultado el 19 de junio de 2020.
} 


\section{EL DERECHO AL TRABAJO Y AL OCIO. DOS TEMAS RELEVANTES EN LA IGUALDAD DE GÉNERO EN ECUADOR}

El derecho al trabajo es un derecho humano "que permite la realización de otros derechos como el de vida digna". ${ }^{31} \mathrm{Y}$ aunque ha existido "un cambio importante a escala global en los últimos cien años ha sido el aumento en la participación de las mujeres en la fuerza de trabajo y en el empleo remunerado $[. .$.$] en la práctica, todavía no se ha logrado la equidad de género en el$ mercado de trabajo". ${ }^{32}$ Las mujeres todavía se enfrentan a un "andamiaje institucional (que) se arraiga una y otra vez en las mujeres, convirtiéndolas en el sujeto prioritario de responsabilidades y derechos relativos a la conciliación, y con el mercado formal", ${ }^{33}$ lo que dificulta que los actores del cambio asuman su papel en la transformación de las relaciones sociales/familiares de género.

Esto a pesar de que el Estado ecuatoriano debe garantizar a las personas trabajadoras el pleno respeto a su dignidad, una vida decorosa, remuneraciones y retribuciones justas, y el desempeño de un trabajo saludable y libremente escogido o aceptado. ${ }^{34} \mathrm{Al}$ respecto, la Corte Constitucional estableció que el derecho al trabajo es un derecho social y económico, ${ }^{35}$ y que está inexorablemente relacionado con la materialización de otros derechos constitucionales, como el derecho a la vida digna, vivienda o los derechos de libertad, ya que permite un desarrollo integral del trabajador, tanto en una esfera particular como en una dimensión social. Es decir, el trabajo se constituye como fuente de ingresos económicos y como fuente de realización personal y profesional; el cual, a su vez, permite al trabajador materializar su proyecto de vida y el de su familia. ${ }^{36}$

Si bien entre los años 2015 y 2019 en América Latina y el Caribe las tasas de participación y ocupación femenina crecieron $(1.8 \%$ y $0.8 \%)$ respectivamente, por efectos de la pandemia estos indicadores han sufrido retrocesos,

31 May Cantillano, Hubert, "El derecho constitucional al trabajo", Boletín Jurídico Virtual IUS Doctrina, núm. 6, 2011, p. 117; disponible en https://revistas.ucr.ac.cr/index.php/iusdoctrina/article/ view/13601.

32 OIT, Panorama laboral temático. Mujeres en el mundo del trabajo. Retos pendientes hacia una efectiva equidad en América Latina y el Caribe, Oficinal Regional para América Latina y el Caribe, p. 22.

33 Faur, Eleonor, "Género, masculinidades y políticas de conciliación familia-trabajo", Nómadas, núm. 24, 2006, p. 132.

34 Constitución de la República del Ecuador, 2008.

35 Corte Constitucional del Ecuador, Sentencia núm. 016-13-SEP-CC, caso núm. 1000-12-EP.

36 Corte Constitucional del Ecuador, Sentencia núm. 241-16-SEP-CC, caso núm. 1573-12-EP. 
siendo el más significativo la baja de $10.4 \%$ en la participación laboral. Según el Informe Laboral 2020 realizado por la OIT, incluso pueden extenderse más allá de la crisis sanitaria sin los soportes de los sistemas públicos de cuidados (enfermos, ancianos, niños), así como el sistema escolar presencial, afectando también la futura reinserción en el mercado laboral. ${ }^{37}$

$\mathrm{Al}$ referirnos al ocio, en el contexto que analizamos estamos tratando el tiempo libre, el tiempo que se dedica al descanso o a la realización de una tarea no obligatoria más bien placentera. En contraposición, la palabra que representa la negación del ocio es "negocio", que proviene del latín nec otium cuya traducción se reconoce como "sin ocio" o "no ocio". Es decir, el derecho al ocio comporta la facultad que tiene una persona para suspender sus tareas productivas y dedicarse al descanso o a la realización de actividades que le generen placer.

De todos los derechos en el marco constitucional ecuatoriano, probablemente el derecho al ocio, que forma parte de la vida digna, es el menos reconocido. La carga emocional negativa que se establece alrededor de la palabra "ocio", genera ideas tales como pereza, holgazanería, vagancia, etc., con lo que parece pasarse por alto que se trata no sólo de un derecho constitucional, sino además de un derecho humano cuyo pleno ejercicio garantiza la protección de otros derechos tales como la salud, el desarrollo de la capacidad creativa, la realización de actividades espirituales, entre otras.

En el contexto internacional de los derechos humanos, este derecho está plenamente reconocido. Es así que la Declaración de los Derechos Humanos se refiere al ocio en su artículo 24, al señalar que "Toda persona tiene derecho al descanso, al disfrute del tiempo libre, a una limitación razonable de la duración del trabajo y a vacaciones periódicas pagadas" (Naciones Unidas, 1948). Por su lado, el PIDESC del 29 de septiembre de 1967, contiene en su artículo 7o. literal d) el compromiso de los Estados parte en el reconocimiento del derecho de toda persona al goce de condiciones de trabajo equitativas y satisfactorias que aseguren, entre otros, el descanso, el disfrute del tiempo libre, la limitación razonable de las horas de trabajo y las vacaciones periódicas pagadas, así como la remuneración de los días festivos.

En el ámbito regional, la Declaración Americana de los Derechos y Deberes del Hombre, en su artículo 15 señala a su vez que "toda persona tiene derecho al descanso, a honesta recreación y a la oportunidad de emplear útilmente el tiempo libre en beneficio de su mejoramiento espiritual, cultural y físico". De conformidad con lo señalado, se evidencia la importancia de este derecho humano que debe ser considerado también, en su relación con otros factores que

37 OIT, Panorama Laboral 2020..., cit., p. 37. 
generan impacto, tal como la cuestión etaria. Así, los niños y las niñas tienen derecho al descanso y el esparcimiento, al juego y a las actividades recreativas propias de su edad, y a participar libremente en la vida cultural y en las artes; y las personas adultas mayores tienen derecho al descanso vinculado con la jubilación. En lo que se refiere al Ecuador, la Constitución contiene varias normas que se orientan hacia el ejercicio del derecho al ocio, como el artículo 24 que establece el derecho de las personas a la recreación y al esparcimiento, a la práctica del deporte y al tiempo libre; y el artículo 66 numeral 2 que reconoce y garantiza el derecho a una vida digna que asegure el trabajo, empleo, descanso y ocio.

Visto así, el derecho al ocio se orienta a una acción o inacción libre y satisfactoria para las personas, no debería tener la connotación negativa que se le reconoce. Sin embargo, la Constitución del Ecuador, en función de su pluralidad inherente, incurre en una contradicción en cuanto al término ocio. Esto debido a que se refiere inicialmente a éste como derecho que configura la vida digna junto a la salud, la alimentación, el agua, la vivienda, el saneamiento ambiental, la educación, el trabajo, entre otros, de conformidad con el artículo 66 numeral 2. Mientras que el artículo 83 numeral 2 del texto constitucional categoriza al ocio como prohibición en cuanto constituye un incumplimiento de los deberes ciudadanos, ordenándose a las ecuatorianas y ecuatorianos a "no ser ociosos", traducción del término quichua ama killa.

Siendo que el ocio se refiere a un momento de satisfacción o disfrute, éste en tanto forma de placer no es accesible a toda la población de la misma forma. De hecho, el ejercicio del ocio como derecho está limitado por factores como el tiempo y/o el dinero, lo que determina que, por ejemplo, la posición económica, la edad, el sexo o el género se conviertan en limitantes para su pleno goce. En lo que se refiere a las mujeres, no es ajeno al conocimiento general que ellas encuentran menos oportunidades de ejercer el derecho al ocio, tanto si se encuentran insertas en el mercado laboral, ejecutando un trabajo remunerado como si no, e incluso estas oportunidades son aún más limitadas en el caso de las mujeres con vulnerabilidades adicionales, tales como las mujeres pobres, migrantes, jefas de hogar, entre otras.

Las labores domésticas o de cuidado que asumen las mujeres, debido a una construcción cultural, cubren casi totalmente su tiempo libre. Algunos autores inclusive establecen que las mujeres ven limitadas sus experiencias de ocio debido a sentimientos de culpa, sumisión, y en general por la satisfacción del deber de cuidar, establecido por la cultura patriarcal como un deseo propio y una necesidad social, ${ }^{38}$ a esto le debemos añadir lo referido

38 Lagarde, Marcela, Mujeres cuidadoras: entre la obligación y la satisfacción, Emakunde, 2003. 
en líneas anteriores, que es el cierre de los centros de cuidado, que tienen como resultado el agravamiento de la situación de las mujeres en este contexto en momentos de pandemia. Resulta útil resaltar, además, lo que señala María Ureta Sosa respecto de las razones que limitan en las mujeres el tiempo de ocio:

...el cuidado de niños, niñas y de otro tipo de dependientes (enfermos o personas adultas mayores discapacitadas) contiene una gran carga ética, moral y subjetiva. Para las mujeres hacerse cargo de los cuidados de menores en el hogar es parte de lo que se espera de ellas, el mandato les impone que cubran las necesidades de otros, no importa si esto implica dejar de lado sus propias necesidades. (...) Este concepto trae consigo implícita la idea de inferioridad, de súbdita y de no merecer tener un tiempo propio o necesidades personales, por ejemplo, la necesidad/derecho de ocio. ${ }^{39}$

De ahí que resulte indispensable que este derecho sea plenamente ejercitado tanto por hombres y mujeres, para contribuir a la erradicación de la desigualdad de género, y que hoy con los efectos de la pandemia se han visto exacerbados. En este sentido, el Consejo Nacional para la Igualdad de Género considera que, en la crisis sanitaria, el aislamiento social ha incrementado las tareas y responsabilidad en el hogar por parte de las mujeres, contando con menos tiempo para actividades de desarrollo personal, profesional y productivo. ${ }^{40}$

\section{DiSCUSIÓN Y RESULTADOS}

En materia de salario, el artículo 2.1 del Convenio número 100 de la OIT desarrolló el principio de igualdad de remuneración y de prohibición de discriminación en salarios. ${ }^{41}$ Según ONU Mujeres, en todo el mundo las mujeres

39 Ureta Sosa, María Ximena, "Roles tradicionales de género y constricciones de ocio en mujeres. Una propuesta de resistencia a las injusticias sociales desde las experiencias de ocio", OcioGune. Ocio e innovación social. Hacia un ocio comprometido con el desarrollo bumano, 2011, p. 57; disponible en https:// www.academia.edu/28273426/Roles_tradicionales_de_género_y_constriccio nes_de_ocio_en_mujeres._Una_propuesta_de_resistencia_a_las_injusticias_sociales_desde_las_experien cias_de_ocio.

40 Consejo Nacional para la Igualdad de Género, Lineamientos del Consejo Nacional para la Igualdad de Género frente a la Crisis Sanitaria, para su Implementación en las Instituciones del Estado, 2020.

41 Convenio sobre Igualdad de Remuneración, núm. 100, 1951. 
apenas ganan 77 centavos por cada dólar que gana un hombre. ${ }^{42}$ Esto provoca una desigualdad de ingresos entre mujeres y hombres de por vida y hace que más mujeres sean abocadas a la pobreza. ${ }^{43}$ "La brecha de género en las remuneraciones de América Latina y el Caribe es del 19\%, comparado con el 24\% a escala mundial". ${ }^{44}$

A pesar de que de manera amplia el derecho a la igualdad y al trabajo son contemplados en la normativa internacional, "la economía feminista ha demostrado que en la dinámica del mercado de trabajo se manifiestan las desigualdades de género". ${ }^{45}$ En este sentido, la brecha salarial de género se mantiene justo por debajo del 15\% en todo el mundo y las mujeres ganan menos que los hombres, ${ }^{46}$ siendo las diferencias salariales entre hombres y mujeres una forma persistente de desigualdad de género, ya que ningún país ha alcanzado la paridad en lo correspondiente a salarios. ${ }^{47}$ A esto se suma la marcada división sexual del trabajo, y "la desigualdad salarial de género constituye una de las problemáticas económicas más analizadas por la literatura feminista". 48

En el contexto ecuatoriano, el Plan Nacional de Desarrollo. Toda una Vida, establece una doble problemática sobre este aspecto, como es la no remuneración igual a las personas por un mismo trabajo y, por otro lado, la existencia de estructuras laborales que afectan de manera diferenciada a hombres y mujeres, presentándose casos en los que las mujeres realizan dobles o triples jornadas para aumentar sus ingresos. ${ }^{49}$

42 ONU Mujeres-Sede, Una Remuneración Igual por Trabajo de Igual Valor; disponible en bttps:// www.unwomen.org/es/news/in-focus/csw61/equal-pay.

43 Idem.

44 El Progreso de las Mujeres en el Mundo 2015-2016. Transformar las economías para realizar los Derechos; disponible en https:// wnw.unwomen.org/-/media/ headquarters/attachments/ sections/library/publications/2015/pown-2015-factsheet-latinamericacaribbean-es.pdf?la =esévs=1541.

45 Ascencio, Débora et al., "Desigualdades de género en el mercado de trabajo argentino: salarios, empleo e informalidad laboral en las mujeres, 2004-2016", Revista de Ciencias Sociales, Universidad de Costa Rica, núm. 165, 2019, p. 80. Pineda A, Javier A., "Trabajo de cuidado: mercantilización y desvalorización”, Revista CS, 2018, p. 117; disponible en https://doi. org/10.18046/recs.iespecial.3218.

46 OECD, The Pursuit of Gender Equality, París, 2017; disponible en bttps://doi. org/10.1787/9789264281318-en.

47 Foro Económico Mundial, Insight Report Global Gender Gap Report 2020, 2019; disponible en bttp:// wmw3.weforum.org/docs/WEF_GGGR_2020.pdf.

48 Ascencio, Débora et al., op. cit., p. 86.

49 Secretaría Nacional de Planificación y Desarrollo, Plan Nacional de Desarrollo 20172021-Toda una Vida, 2017; disponible en http://mmw.planificacion.gob.ec/wp-content/ uploads/down loads/2017/10/PNBV-26-OCT-FINAL_OK.compressed1.pdf. 
Esta realidad se muestra aún más compleja, ya que la OIT estima que la crisis por la pandemia causará una reducción del 10.3\% de las horas de trabajo, según las cuales afectará mayormente a América en 12.4\%, y Europa y Asia Central en $11.8 \%$ de pérdida de horas de trabajo. ${ }^{50}$ Lamentablemente, a la reducción de horas de trabajo hay que añadir que "durante los eventos de contracción económica que América Latina y el Caribe ha registrado a través de su historia, el mercado laboral de la región experimentó aumentos en la informalidad del empleo". ${ }^{51}$

En lo correspondiente a la brecha en el ingreso laboral promedio, el gráfico 1 , con datos a valores reales a diciembre de 2007, nos muestra que, en diciembre de 2010, el ingreso laboral promedio de un hombre que posee empleo fue de 329.60 dólares; en cambio, las mujeres obtuvieron un ingreso de 268.30 dólares. Por otro lado, a diciembre de 2019, se observa una leve mejoría en esta brecha, llegando a los 345.50 dólares en el promedio de los hombres y a los 292.70 dólares en el caso de las mujeres; es decir, en el periodo señalado existió una leve mejoría. Por cada dólar que ganaba un hombre, las mujeres pasaron de ganar 81 centavos a 85 centavos, situación que si bien muestra un incremento, evidencia de forma clara la desigualdad salarial entre hombres y mujeres.

\section{GRÁFICO 1. INGRESOS LABORALES POR SEXO \\ ECUADOR}

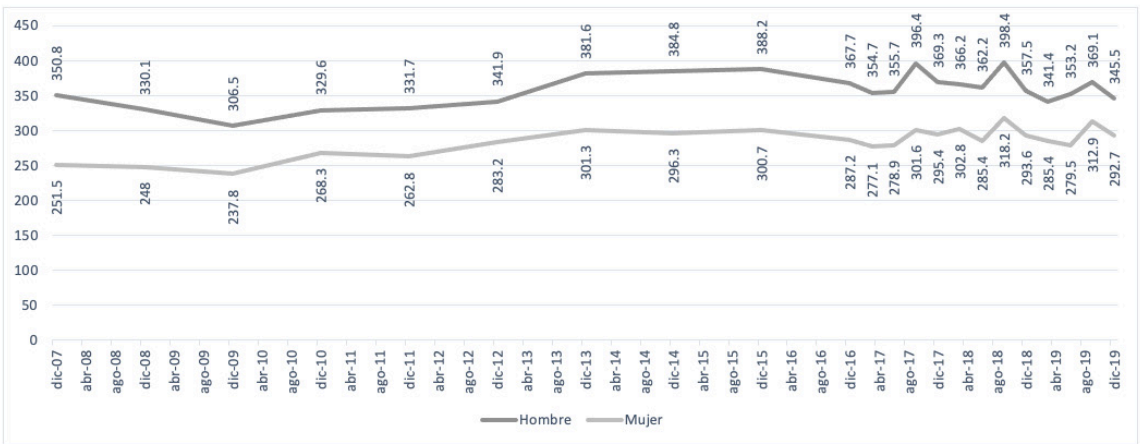

FUENTE: INEC-ENEMDU. Elaboración propia.

50 OIT, Observatorio de la OIT: El COVID-19 y el mundo del trabajo. Tercera edición; disponible en: https:// wmw.ilo.org/wcmsp5/groups/public/---dgreports/---dcomm/documents/briefingnote/woms_743154.pdf.

51 Organización Internacional del Trabajo OIT, Panorama laboral temático..., cit., p. 46. 
Se observa que las brechas entre los salarios se mantienen a pesar de que en varios Estados existen salarios mínimos establecidos por ley. En el 2020 algunos países efectuaron incrementos en los salarios mínimos, situación distinta ocurrida al Ecuador, en donde para 2021 se mantuvo el salario básico de 400 dólares de los Estados Unidos de América, a pesar de que en años anteriores éste se vio incrementado.

\section{GRÁFICO 2. SALARIO BÁSICO \\ ECUADOR (2010-2021)}

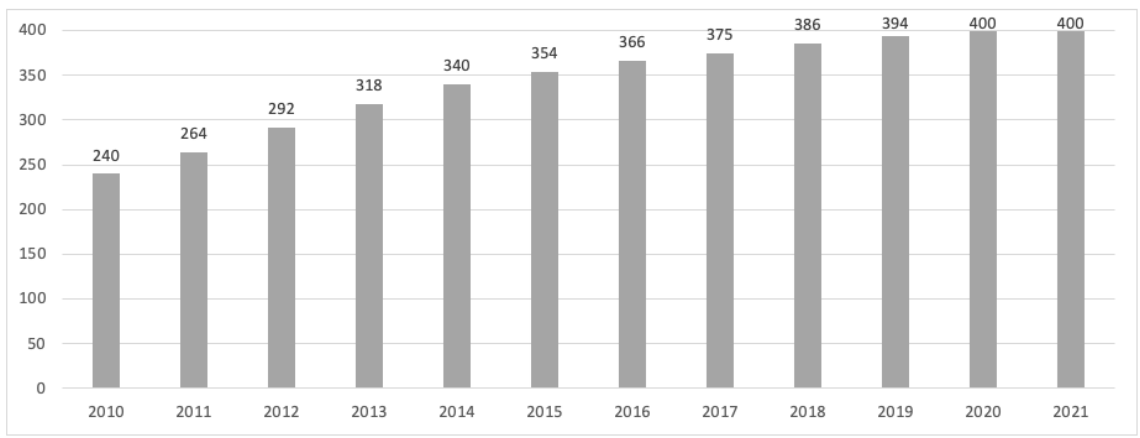

FUENTE: INEC-ENEMDU. Elaboración propia.

$\mathrm{Al}$ abordar el tema de las mujeres, nos damos cuenta de que "en todas las regiones, la proporción de mujeres es mayor entre quienes perciben el salario mínimo a un monto inferior que entre quienes perciben un monto superior al del salario mínimo". ${ }^{52}$ Situación que lleva a que quienes realizan trabajos agrícolas y trabajo doméstico, sean los grupos que con más frecuencia quedan excluidos de la cobertura legal de los sistemas de salario mínimo. ${ }^{53}$

La OIT insta por la adopción de respuestas políticas urgentes y significativas para proteger tanto a las empresas, en particular las más pequeñas, como a los trabajadores, sobre todo aquellos que operan en la economía informal. ${ }^{54}$ Esto se debe a que más de 2000 millones de personas en el mundo trabajan en la economía informal, y por lo tanto no cuentan con

52 OIT, Informe Mundial sobre Salarios 2020-2021. Los Salarios y el Salario Mínimo en Tiempos de la COVID-19. Resumen Ejecutivo, Suiza, 2020, p. 4.

53 Ibidem, p. 3.

54 OIT, Observatorio de la OIT..., cit. 
protección como la seguridad social, situación que deriva a un acceso limitado a los servicios de atención de la salud y también a no poseer ingresos en caso de enfermedad o confinamiento. ${ }^{55}$ América Latina y el Caribe son regiones en donde una de cada dos personas trabajadoras tiene un empleo informal ${ }^{56} \mathrm{y}$ según las proyecciones para 2021 existe incertidumbre, particularmente por los efectos asociados a segundas olas y rebrotes de la pandemia de la COVID-19, que impliquen nuevas medidas de confinamiento, reducción de la movilidad de las personas y sus consecuentes efectos en la actividad económica, la participación laboral y el empleo. ${ }^{57}$

Entonces, el desarrollo de estas medidas afectará de sobremanera la posibilidad del ejercicio del empleo informal, ya que no pueden realizarse actividades como el teletrabajo. El trabajo informal en las mujeres tiene grandes impactos, ya que el $42 \%$ de los trabajadores de este sector son mujeres, frente al 32\% que representan los hombres, trayendo consigo un incremento de la tasa de pobreza y por tanto su perpetuación..$^{58}$ Esta situación de desigualdad la podemos también observar en las tasas de empleo global (véase gráfico 3).

\section{GRÁFICO 3. TASA DE EMPLEO GLOBAL POR SEXO \\ ECUADOR}

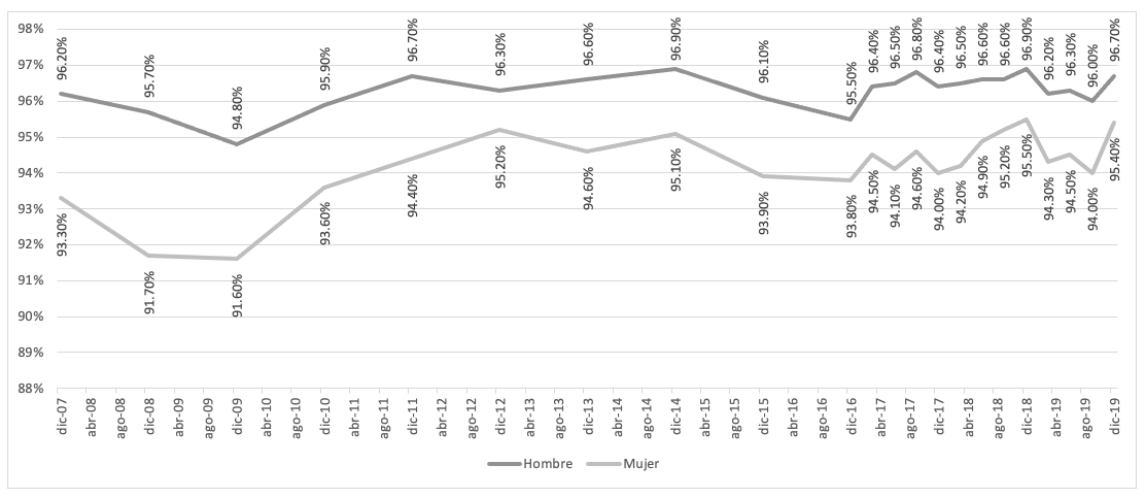

FUENTE: INEC-ENEMDU. Elaboración propia.

$\begin{array}{ll}55 & \text { Idem. } \\ 56 & \text { Sánchez y Torres, op. cit., p. } 2 . \\ 57 & \text { OIT, Panorama Laboral 2020..., cit., p. } 58 . \\ 58 & \text { OIT, Observatorio de la OIT..., cit. }\end{array}$


Para el año 2010, los hombres se ubicaron en el 95.9\%, pero, la tasa de las mujeres se ubicó en un $93.6 \%$, siendo ésta 2.3 puntos porcentuales menor que la de los hombres. En lo correspondiente a la tasa de empleo, existe una mejoría para diciembre de 2019 en donde se reduce a 1.3 puntos porcentuales, ubicándose en $96.7 \%$ para los hombres y $95.4 \%$ para las mujeres.

Esta brecha se profundiza al analizar la tasa de empleo adecuado/pleno por sexo (véase gráfico 4); si observamos diciembre de 2010, para los hombres se tiene una tasa de $50.0 \%$ y de $36.5 \%$ para las mujeres, existiendo una diferencia de 13.5 puntos porcentuales a favor de los hombres.

Ahora bien, si se tiene en cuenta el contexto de la pandemia, en septiembre de 2020 (con una muestra menor a periodos anteriores a la pandemia) la tasa de los hombres con empleo adecuado/pleno cae a un valor del $36.20 \%$, y las mujeres cuentan con una tasa del $26.40 \%$ el cual es un valor en extremo reducido; en este caso, la diferencia porcentual entre sexos es de casi diez puntos.

En este gráfico, es clara la caída de las tasas para el periodo mayo/junio de 2020, esto puede explicarse debido a los efectos de la pandemia, pero también se debe al cambio de metodología muestral empleada para el levantamiento de datos, la cual fue llevada a través de una encuesta telefónica en donde los estratos más empobrecidos tuvieron menor representación.

\section{GRÁFICO 4. TASA DE EMPLEO ADECUADO/PLENO POR SEXO \\ ECUADOR}

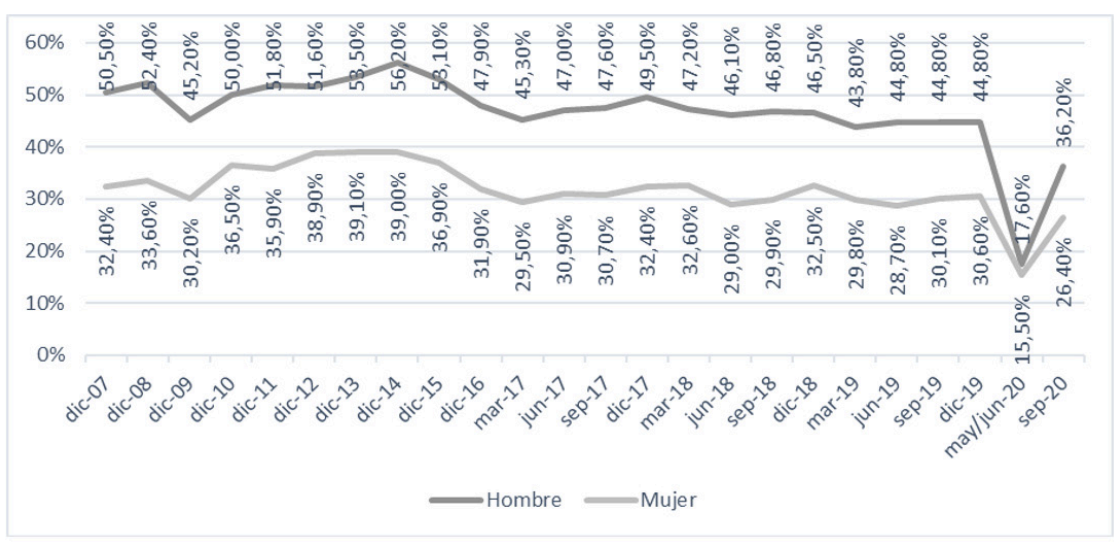

FUENTE: INEC-ENEMDU. Elaboración propia. 
Dentro del mismo periodo, respecto al indicador de subempleo (véase gráfico 5), la tasa en diciembre de 2010 para los hombres se ubicó en $14.1 \%$ y para las mujeres en $13.4 \%$, por lo cual existió menos de un punto porcentual de diferencia entre sexos; y para septiembre de 2020, esta diferencia se vio incrementada a 2.7 puntos porcentuales, en donde superaron los hombres a las mujeres.

\section{GRÁfico 5. TASA DE SUBEMPLEO POR SEXO ECUADOR}

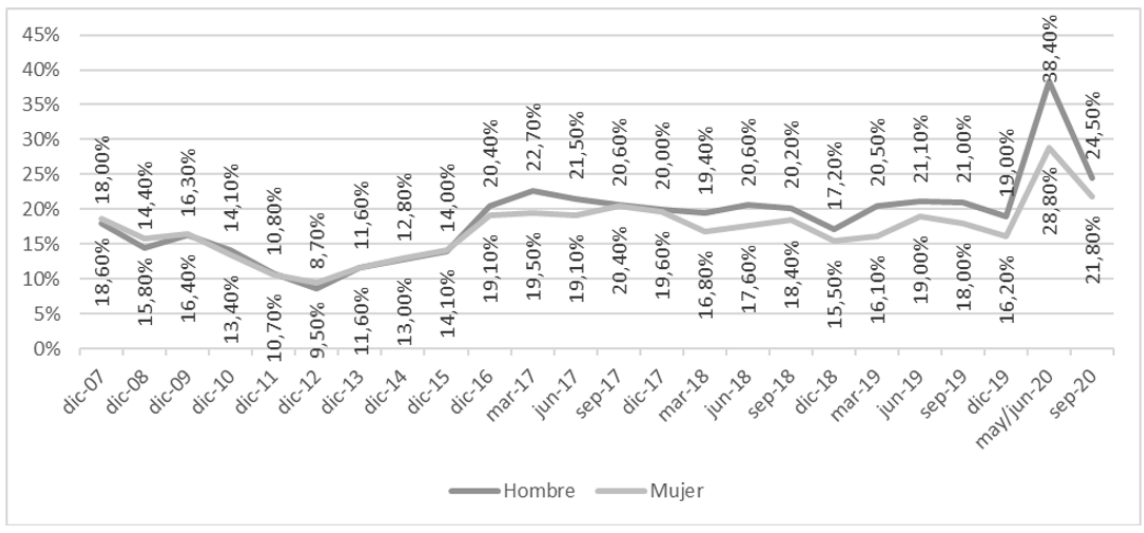

FUENTE: INEC-ENEMDU. Elaboración propia.

Mientras la pandemia continúe y las medidas de aislamiento se apliquen, el efecto del desempleo seguirá incrementándose. ${ }^{59}$ En cuanto a las cifras de desempleo, el gráfico 6 nos señala que, en diciembre de 2010, las mujeres tuvieron una tasa de desempleo del $6.4 \%$, mientras que los hombres una tasa del $4.1 \%$; estos 2.3 puntos porcentuales de diferencia se mantienen aún diez años después y en contexto de pandemia; cabe señalar que entre 2011 y 2019 , la diferencia entre sexos se mantuvo en promedio en un valor inferior a los dos puntos porcentuales: su interpretación es que esta tasa sufrió un impacto negativo en la brecha entre sexos debido a la pandemia sufrida a nivel mundial.

En América Latina aún no existen políticas orientadas al género femenino, esto porque aún persisten las responsabilidades claramente diferenciadas tanto en el trabajo productivo como reproductivo, "donde se promueven la estructuración o el sostenimiento de una determinada matriz de responsabili-

59 CEPAL/OIT, Coyuntura..., cit., p. 7. 
dades de provisión y de cuidado según el sexo, como las representaciones que los sujetos construyen acerca de dicha estructuración". ${ }^{60}$

\section{GRÁFICO 6. TASA DE DESEMPLEO POR SEXO \\ ECUADOR}

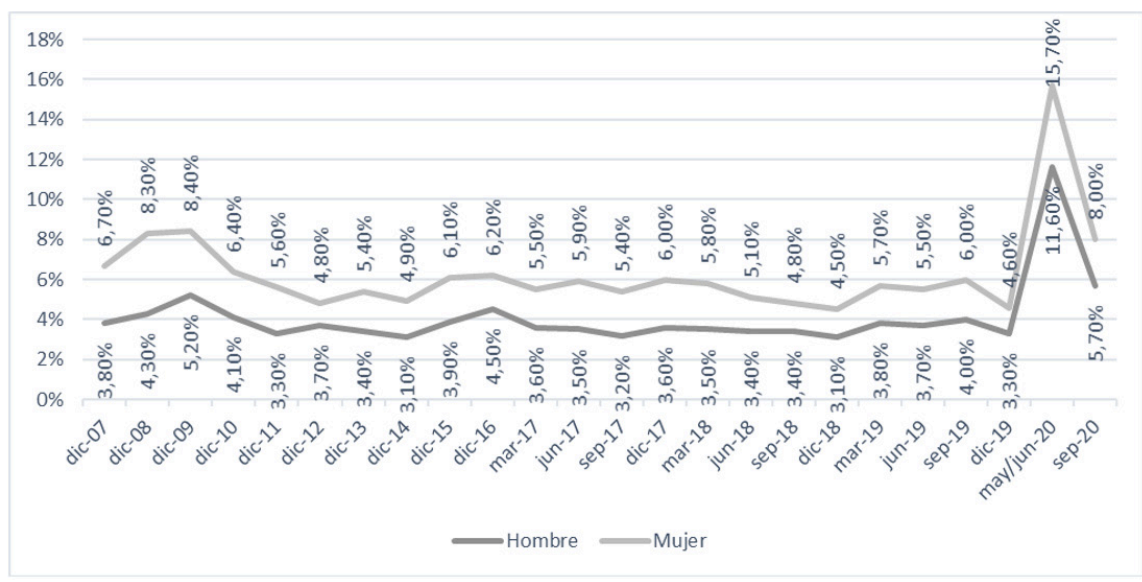

FUENTE: INEC-ENEMDU. Elaboración propia.

Persiste la necesidad de que el Estado ecuatoriano y cada una de sus instituciones busquen claridad en su desarrollo económico y social, el mismo que potencialice el trabajo femenino a través de políticas de género que incrementen la inserción y permanencia de las mujeres en el mercado de trabajo, generando un verdadero imperativo de justicia de género y se logre cerrar la brecha existente que se ha planteado como meta hasta el 2021, reduciendo la brecha de empleo adecuado entre hombres y mujeres, del 33.5\% al 28.7\%, y la brecha salarial entre hombres y mujeres del $21.9 \%$ al $18.4 \%$.

Por otra parte, en lo que respecta a la brecha de género relacionada al derecho al ocio, se observa que a pesar de su reconocimiento constitucional, en el Ecuador, las mujeres, en comparación con los hombres, dedican un menor porcentaje de su tiempo no laboral a asuntos de naturaleza personal. En el caso de las mujeres, el tiempo personal o el tiempo dedicado a actividades no productivas (2012) llega a un valor del 54\%; mientras que en el caso de los hombres llega al 60\% (véase gráfico 7).

60 Faur, op. cit., p. 130. 


\section{GRÁFICO 7. DISTRIBUCIÓN DEL TIEMPO TOTAL POR SEXO: POBLACIÓN OCUPADA DE 12 AÑOS Y MÁS \\ ECUADOR}

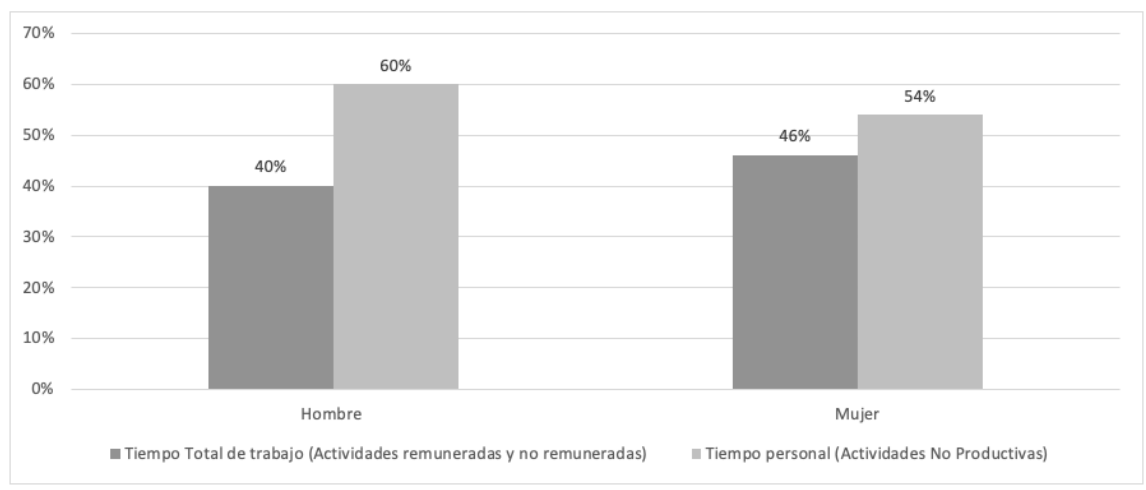

FUENTE: INEC-EUT. Elaboración propia.

\section{GRÁFICO 8. TIEMPO ACTIVIDADES PERSONALES-HORAS SEMANALES ECUADOR}

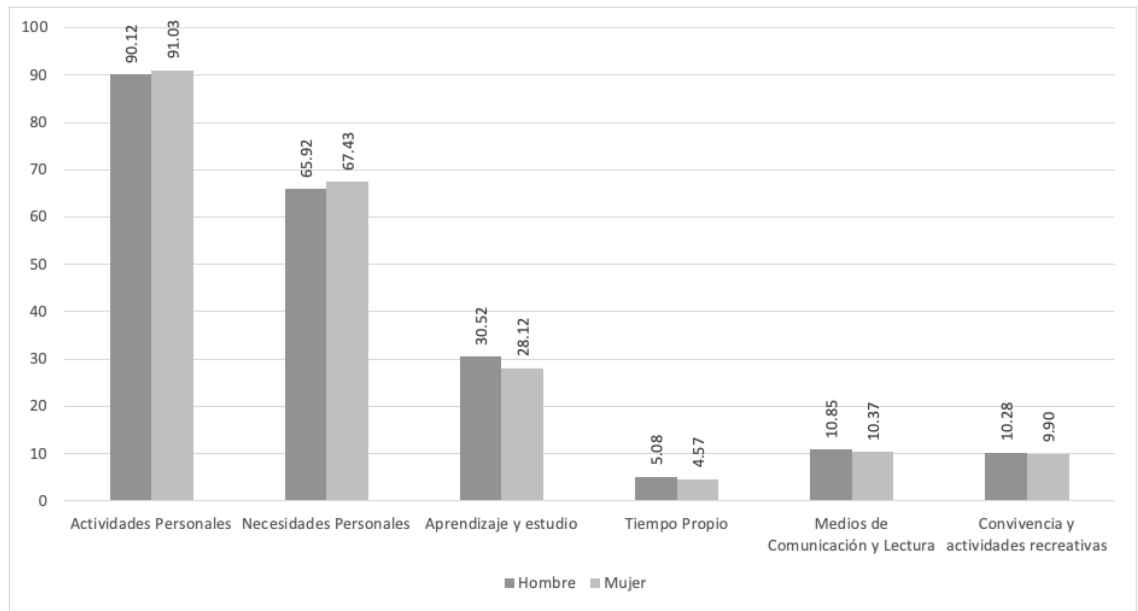

FUENTE: INEC-EUT. Elaboración propia. 
En la misma línea, en cuanto al manejo del tiempo personal, en horas promedio a la semana, conforme se desprende del gráfico 8 , las mujeres tienen menos tiempo de dedicación a actividades no laborales de crecimiento particular o placer, tales como el aprendizaje y estudio, tiempo propio, medios de comunicación y lectura, y convivencia y actividades recreativas. En todos estos casos, las mujeres dedican menos horas que los hombres a estas actividades de ocio.

Todos estos datos, respecto de Ecuador, son además un reflejo de lo que sucede en América Latina y en el resto del mundo. Las mujeres no sólo no acceden al mercado laboral con facilidad, y cuando lo hacen, las condiciones no son las mismas de las que gozan sus pares masculinos; además, tampoco tienen las mismas oportunidades para acceder a momentos de ocio o recreación. De esta manera, el trabajo remunerado puede ser un pilar fundamental para la igualdad sustantiva de las mujeres, pero sólo cuando exista una responsabilidad compartida en el trabajo de cuidados no remunerado, ya que esto permitirá a las mujeres disponer de tiempo para el ocio y el aprendizaje. ${ }^{61}$

\section{CONCLUSIONES}

$\mathrm{Al}$ analizar el derecho al trabajo, se observa que es un derecho social y económico que es interdependiente de otros derechos constitucionales, como la vida digna. Esto en cuanto permite a la trabajadora o trabajador lograr un desarrollo integral desde el fuero personal, pero que al mismo tiempo genera un desarrollo sostenible en el modelo económico. No obstante, la realidad nos muestra que en Estados que poseen estructuras estatales y sociales desiguales, el ejercicio de estos derechos no es equitativo entre hombres y mujeres. Estas situaciones se han visto más afectadas por la pandemia de la COVID-19, en la cual las mujeres están sometidas a diversas circunstancias que limitan su pleno acceso al derecho al trabajo o a un trabajo en condiciones dignas.

De manera que el pleno ejercicio del derecho al trabajo en igualdad sólo será posible cuando se reduzcan las diferentes brechas sociales, políticas, culturales y económicas a las que se enfrentan las mujeres en el mundo y en nuestro país. Para que esto ocurra, se deben generar políticas públicas sobre la igualdad entre hombres y mujeres en materia educativa; ya que la educación se correlaciona con los salarios en la medida en que las mujeres menos educadas

\footnotetext{
61 OIT, Panorama laboral temático..., cit.
} 
generalmente ganan menos, como lo manifiesta la OCDE. Por esta razón, organismos como la OIT insisten en la importancia de la educación y formación profesional para el fomento a la igualdad de oportunidades laborales.

Esto expone la real importancia de caminar hacia una transición justa, ya que para el Ecuador el sector de la bioeconomía: agricultura, ganadería, forestal y pesca, representa el 29\% del total de la población ocupada; y en éstas se sostiene importantes desigualdades de género, ya que es un sector con empleo informal, y la principal fuente de ingresos para las personas con menos recursos económicos. Algo similar ocurre en el sector de turismo, que es uno de los principales impulsores del empleo y el crecimiento, ${ }^{62}$ que ha sido impactado por la COVID-19; y se prevé que se contraiga entre el 45\% y $70 \% .{ }^{63} \mathrm{El}$ sector de residuos y economía circular se suma a su alto nivel de informalidad, ${ }^{64}$ en el cual el $27 \%$ de personas ocupadas en la gestión de desechos son mujeres. ${ }^{65}$

Es importante destacar que para reducir las brechas de género en el empleo se debe transitar hacia un equilibrio entre la vida laboral y familiar, así como la distribución equitativa de las responsabilidades laborales no remuneradas promoviendo no sólo la mayor participación laboral femenina, sino a través del ejercicio de los derechos de los trabajadores varones en conciliar las responsabilidades laborales y familiares, así como su participación en la división de las tareas reproductivas y de cuidado.

La desigualdad respecto a las remuneraciones y la dedicación horaria a las tareas de cuidado conducen a otra brecha respecto al derecho al descanso y ocio de las mujeres, quienes en comparación con sus pares masculinos tienen menos horas para aprendizaje y estudio, para actividades de convivencia, e incluso para actividades recreativas o de placer. De ahí que es necesario que el Estado en conjunto con la sociedad civil se preocupen por alcanzar la plena igualdad en el Ecuador entre hombres y mujeres en lo atinente a los derechos al trabajo y al ocio. Con esto se podrá lograr un desarrollo sostenible y el empoderamiento económico como elemento clave para la eliminación de la violencia contra las mujeres.

62 OIT, COVID-19 and the Tourism Sector, 2020; disponible en bttps:/ / wmm.ilo.org/ sector/ Resources/publications/WCMS_741468/lang--en/index.htm.

63 OIT, The Impact of COVID-19 on the Tourism Sector, 2020, p. 2; disponible en bttps:// www.ilo.org/wcmsp5/groups/public/---ed_dialogue/---sector/documents/briefingnote/wcms_741468.pdf.

64 BID, Reciclaje en Tiempos de Pandemia, 2020; disponible en https:/ / blogs.iadb.org/ agual es/reciclaje-en-tiempos-de-pandemia/.

65 Sánchez y Torres, op. cit., p. 10. 


\section{BIBLIOGRAFÍA}

AgUiLÓ RegLA, Josep, "Positivismo y postpositivismo: dos paradigmas jurídicos en pocas palabras", Doxa. Cuadernos de Filosofía del Derecho, núm. 30, 2007; disponible en https:// doi.org/10.14198/doxa2007.30.55.

Asamblea General de NACIONES Unidas, Convención sobre la Eliminación de Todas las Formas de Discriminación Contra la Mujer, 1979.

Asamblea General de NACiOnes Unidas, Transformar nuestro Mundo. La Agenda 2030 para el Desarrollo Sostenible.

ASCENCIO, Débora et al., "Desigualdades de género en el mercado de trabajo argentino: salarios, empleo e informalidad laboral en las mujeres, 20042016", Revista de Ciencias Sociales, Universidad de Costa Rica, 2019.

BID, Reciclaje en Tiempos de Pandemia, 2020; disponible en https://blogs. iadb.org/agua/es/reciclaje-en-tiempos-de-pandemia/, consultado el 11 de enero de 2021.

CAAMAÑO ROJO, Eduardo, "Mujer y trabajo: origen y ocaso del modelo del padre proveedor y la madre cuidadora", Revista de derecho, núm. 34, 2010; disponible en https:// doi.org/10.4067/S0718-68512010000100005.

CARCEDO, Ana et al., Apertura económica, género y pobreza en el istmo centroamericano, 2009; disponible en http:/ / www.gemlac.org/attachments/article/313/Informe septiembre 2009.pdf.

CEPAL/OIT, Coyuntura Laboral en América Latina y el Caribe. El Trabajo en Tiempos de Pandemia: Desafíos frente a la Enfermedad por Coronavirus (COVID-19), Santiago, 2020.

Conferencia Mundial de Derechos Humanos, Declaración y Programa de Acción de Viena, 1993.

COnSEjo Nacional para la Igualdad DE GÉNERO, Lineamientos del Consejo Nacional para la Igualdad de Género frente a la Crisis Sanitaria, para su Implementación en las Instituciones del Estado, 2020.

CONSTITUCiÓn de la República del Ecuador, 2008.

CONVENIO sobre Igualdad de Remuneración, núm. 100, 1951; disponible en https:// mwn.ilo.org/dyn/normlex/es/f?p=NORMLEXPUB:12100:0::NO: 12100:P12100_INSTRUMENT_ID:312245:NO.

CORTE CONSTITUCiONAL DEL ECUAdOR, Sentencia núm. 292-16-SEP-CC emitida en el caso núm. 0734-13-EP. 
Corte COnstitucional Del ECuAdor, Sentencia núm. 016-13-SEP-CC, caso núm. 1000-12-EP.

CORTE CONSTITUCIONAL DEL ECUAdOR, Sentencia núm. 241-16-SEP-CC, caso núm. 1573-12-EP.

EL Progreso de las Mujeres en el Mundo 2015-2016. Transformar las Economías para realizar los Derechos; disponible en https://www.unwomen. org/-/ media/ headquarters/attachments/sections/library/publications/2015/poww2015-factsheet-latinamericacaribbean-es.pdf?la $=$ es \& vs $=1541$.

Eusko JaurlaritZa Gobierno VASCO et al., Tribunal Internacional de Derechos de las Mujeres. Viena +20. Euskalherria 2013, Mugarikgab, 2013; disponible en wmw.mugarikgabe.org.

FACio, Alda y Victoria, Anya, "Los derechos culturales y los derechos de las mujeres son derechos humanos y como tales, deben ser gozados en igualdad", Revista Europea de Derechos Fundamentales, núm. 29, 2017; disponible en https:/ / dialnet.unirioja.es/servlet/articulo?codigo $=6143999$.

FAUR, Eleonor, "Género, masculinidades y políticas de conciliación familiatrabajo", Nómadas, núm. 24, 2006; disponible en bttps://doi.org/10.1787 19789264281318-en.

Foro ECONÓMico Mundial, Insight Report Global Gender Gap Report 2020, 2019; disponible en http://www3.weforum.org/docs/WEF_GG GR_2020.pdf.

GIL ACEVEDO, Juan Carlos, "Ruta hacia la dignificación laboral de las madres comunitarias y sustitutas en Villavicencio, Colombia", Estudios de Derecho, núm. 72, 2015; disponible en bttps://doi.org/10.17533/udea.esde. v72n160a07.

Instituto Nacional de Estadísticas y Censos, Atlas de Género, 2018; disponible en https:// wwn.ecuadorencifras.gob.ec/documentos/web-inec/Bibliotecas /Libros/Atlas_de_Genero_Final.pdf.

LAGARDE, Marcela, Mujeres cuidadoras: entre la obligación y la satisfacción, Emakunde, 2003.

May Cantillano, Hubert, "El derecho constitucional al trabajo", Boletín Jurídico Virtual IUS Doctrina, núm. 6, 2011; disponible en bttps:/ / revistas.ucr. ac.cr/index.php/iusdoctrina/article/view/13601.

NACIONES UnIDAS, Declaración Universal de Derechos Humanos, 1948.

NACIONES UNIDAS, The Impact of COVID-19 on Women, 2020; disponible en bttps:// www.unwomen.org/-/ media/ headquarters/attachments/sections/ 
library/publications/2020/policy-brief-the-impact-of-covid-19-on-women-en. pdf?la = enevs $=1406$.

NAVArro, Marysa, "Los derechos humanos de las mujeres", Foro de la Filantropía y Mujeres, México, 1993.

OECD, The Pursuit of Gender Equality, París, 2017; disponible en bttps:// doi.org/10.1787/9789264281318-en.

OIT, Brindar Apoyo a los Ingresos y al Empleo en Tiempos de la COVID-19: Articular las Transferencias en Efectivo con Políticas Activas del Mercado de Trabajo (PAMT).

OIT, COVID-19 and the Tourism Sector, 2020; disponible en bttps:/ / www.ilo. org/sector/Resources/publications/WCMS_741468/lang--en/index.htm.

OIT, Informe Mundial sobre Salarios 2020-2021. Los Salarios y el Salario Mínimo en Tiempos de la COVID-19. Resumen Ejecutivo, Suiza, 2020.

OIT, Observatorio de la OIT: El COVID-19 y el Mundo del Trabajo. Tercera edición; disponible en bttps://www.ilo.org/womsp5/groups/public/---dgreports/--dcomm/documents/briefingnote/woms_743154.pdf.

OIT, Panorama Laboral 2020. América Latina y el Caribe, 2020.

OIT, Panorama laboral temático. Mujeres en el mundo del trabajo. Retos pendientes hacia una efectiva equidad en América Latina y el Caribe, Oficinal Regional para América Latina y el Caribe.

OIT, The Impact of COVID-19 on the Tourism Sector, 2020; disponible en bttps:// www.ilo.org/womsp5/groups/public/---ed_dialogue/---sector/documents/ briefingnote/woms_741468.pdf.

OIT y BID, Jobs in a Net-Zero Emissions Future in Latin America and the Caribbean, 2020; disponible en bttp:/ / dx.doi.org/10.18235/0002509.

ONU Mujeres-Sede, Una Remuneración Igual por Trabajo de Igual Valor; disponible en bttps:// wnw.unwomen.org/es/news/in-focus/csw61/ equal-pay.

PINEDA D., Javier A., "Trabajo de cuidado: mercantilización y desvalorización”, Revista CS, 2018; disponible en https://doi.org/10.18046/recs.iespe cial.3218.

QuÉ Hacemos. Empoderamiento Económico, ONU Mujeres, América Latina y el Caribe; disponible en https://lac.unwomen.org/es/que-bacemos/empode ramiento-economico.

SÁnCHEZ, Ana Belén y TORRES, Braulio, Una recuperación verde y justa en América Latina y el Caribe: una perspectiva desde el mundo del trabajo, OIT, 2020. 
Secretaría Nacional De Planificación Y Desarrollo, Plan Nacional de Desarrollo 2017-2021-Toda una Vida, 2017; disponible en bttp:/ / www.planificacion.gob.ec/wp-content/ uploads/downloads/2017/10/PNBV-26OCT-FINAL_OK.compressed1.pdf

TOMEI, Manuela, y VegA-Ruiz, María Luz, "La discriminación de la mujer en el lugar de trabajo. Nuevas tendencias en materia de discriminación por motivos basados en la maternidad y el acoso sexual", Revista Latinoamericana de Derecho Social, núm. 4, 2007; disponible en http:/ / biblio.juridicas.unam. $m x /$ revista/pdf/DerechoSocial/4/art/art8.pdf.

URETA Sosa, María Ximena, "Roles tradicionales de género y constricciones de ocio en mujeres. Una propuesta de resistencia a las injusticias sociales desde las experiencias de ocio", OcioGune. Ocio e innovación social. Hacia un ocio comprometido con el desarrollo bumano, 2011; disponible en https: / wmw.aca demia.edu/28273426/Roles_tradicionales_de_género_y_constricciones_de_ocio_en_ mujeres._Una_propuesta_de_resistencia_a_las_injusticias_sociales_desde_las_expe riencias_de_ocio.

VAlenZuela Reyes, María Delgadina, "Evolución legislativa sobre los derechos e igualdad jurídica de la mujer en México", Revista Latinoamericana de Derecho Social, núm.10, 2010. 